\title{
Free Energy Simulations of Cargo-Carrier Interactions for Core-Multishell Nanotransporters
}

\author{
Marcus Weber ${ }^{1}$, Christian Zoschke ${ }^{2}$, Amir Sedighi ${ }^{1}$, Emanuel Fleige $^{3}$, Rainer Haag ${ }^{3}$ and Monika Schäfer-Korting ${ }^{2 *}$ \\ ${ }^{1}$ Zuse Institute Berlin (Computational Molecular Design), Berlin, Germany \\ ${ }^{2}$ Institute for Pharmacy (Pharmacology and Toxicology), Freie Universität Berlin, Berlin, Germany \\ ${ }^{3}$ Institute of Chemistry and Biochemistry (Organic Chemistry), Freie Universität Berlin, Berlin, Germany
}

\begin{abstract}
Dendritic core-multishell (CMS) nanotransporters are composed of three parts: a polyglycerol amine core covalently linked to an inner alkyl shell and an outer polyethylene glycol shell. Aiming to unravel the preferred localization of the guest molecule within the locally well-tolerated delivery system, we transferred molecular dynamics simulations to CMS nanotransporters and verified the results with experimental data. Differences in free energy of the planar, nonpolar, and lipophilic Nile red ( $\log P$ 3.4) indicated a preferential location within the inner CMS nanotransporter shell. Differences in free energy of the globular, polar and hydrophilic morphine $(\log P \leq 0.8)$ predicted poor loading which has been verified. Replacing the outer CMS nanotransporter shell by glutamate or aspartate results in electrostatic forcemediated morphine attachment. Thus, the investigation of larger molecular systems consisting of many similar building blocks becomes feasible with our approach. In conclusion, the computational approach based on differences in free energy may improve the design of tailor-made CMS nanotransporters and enhance drug development.
\end{abstract}

Keywords: Dendritic core-multishell nanotransporters; Drug delivery system; Molecular dynamics simulations; Morphine; Nile red

Abbreviations: CMS: Dendritic Core-Multishell; MD: all-atomic Molecular Dynamics; mPEG: Poly(ethylene glycol); PG: Polyglycerol amine

\section{Introduction}

Dendritic core-multishell (CMS) nanotransporters incorporate planar-nonpolar or planar-polar cargo molecules [1-3]. Increasing the notoriously low solubility of lipophilic agents, the CMS nanotransporters offer the potential for well-tolerated for drug delivery [4]. Skin penetration of lipophilic and hydrophilic dyes was significantly enhanced when loaded to CMS nanotransporters and compared to standard formulations [5-7]. CMS nanotransporters are composed of a hyperbranched polyglycerol amine (PG) or polyglycerol core, covalently attached (via the superficial amine / hydroxyl groups) to an inner C18-alkyl chain (dicarboxy acid) and connected to an outer monomethoxy poly (ethylene glycol) shell (mPEG; Figure 2A). Unimers (7-16 nm) aggregate spontaneously to clusters of up to $200 \mathrm{~nm}$ in size.

The early evaluation of drug delivery promotes pre-clinical drug development, however, limited amounts of investigational new drugs available at the beginning of drug development preclude the concomitant development of the drug and its carrier. With nanocarriers of well-defined structure, mathematical approaches can be used in early stages of drug development. All-atomic molecular dynamics (MD) simulations have been recently improved by combining two approaches: correlation of preferential binding modes with free energy differences and a Monte Carlo correlation of preferential binding modes with conformational entropy differences. Improved MD simulations characterize metastable steps of the binding process, yield the corresponding transition probabilities, and is independent of lengthy molecular dynamics trajectories $[8,9]$.

In this first feasibility study, we investigated the preferred location of two guest compounds when loaded to CMS nanotransporters by calculating free energy differences. We adapted the hands-free $\mathrm{MD}$ simulations from ligand-receptor interactions to cargo-carrier simulations. We chose the well-entrapped Nile red (planar-nonpolar,
[2,6]) and morphine (globular-polar, [10]). Moreover, to address the unlikely loading of non-protonated morphine and protonated morphine- $\mathrm{H}+$ to the CMS nanotransporters we tested a structurally altered carrier for improved morphine loading (Figure 1).

\section{Methods}

\section{Adaption of MD simulations for CMS nanotransporters}

Despite of the CMS nanotransporters weight (around $70.000 \mathrm{~g} \mathrm{~mol}$ $\left.{ }^{1}\right)$, we performed all calculations within one week on a 16 CPU machine with 256GB RAM. To reduce the computational power needed for MD simulations of systems with more than ten thousand atoms we derived entropy and inner energy from a cone-shaped substructure which reflects the entire system of the nanoparticles architecture. The movement of guest molecules between core and shells of the CMS nanotransporter, however, is on much larger timescales than used for MD simulations.

We assumed a spherically shaped nanotransporter (radius: $11-13 \mathrm{~nm}$ in total; 3-5 $\mathrm{nm}$ core; 3-5 nm inner shell; 3-4 nm outer shell) as starting point for MD simulations of a CMS nanotransporter. The chemical available nanotransporter is built of a core (molecular weight $10,000 \mathrm{~g}$ $\mathrm{mol}^{-1}$, approximately 70 repeating units) with a degree of amination of $70 \%$ (corresponding to approximately 95 amine groups), all amine groups are functionalized with a $\mathrm{C} 18$ alkyl chain covalently bound by mPEG350 (350 $\mathrm{g} \mathrm{mol}^{-1}$, approximately 6-7 repeating units). Gromacs

*Corresponding author: Monika Schäfer-Korting, Freie Universität Berlin Institute for Pharmacy (Pharmacology and Toxicology), Königin-Luise-Str. 2+4 14195 Berlin, Germany, Tel: 49-30-838-53283; Fax: 49-30-838-470871; E-mail: monika.schaefer-korting@fu-berlin.de

Received August 05, 2014; Accepted October 04, 2014; Published October 14 2014

Citation: Weber M, Zoschke C, SedighiA, Fleige E, Haag R, et al. (2014) Free Energy Simulations of Cargo-Carrier Interactions for Core-Multishell Nanotransporters. J Nanomed Nanotechnol 5: 234. doi: 10.4172/2157-7439.1000234

Copyright: @ 2014 Weber M, et al. This is an open-access article distributed under the terms of the Creative Commons Attribution License, which permits unrestricted use, distribution, and reproduction in any medium, provided the original author and source are credited. 


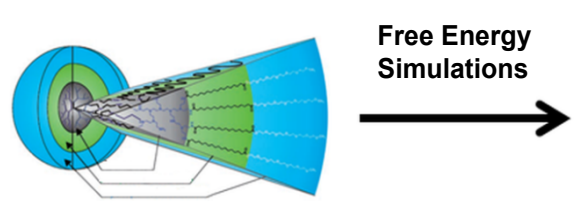

Dendritic Core-Multishell Nanotransporter

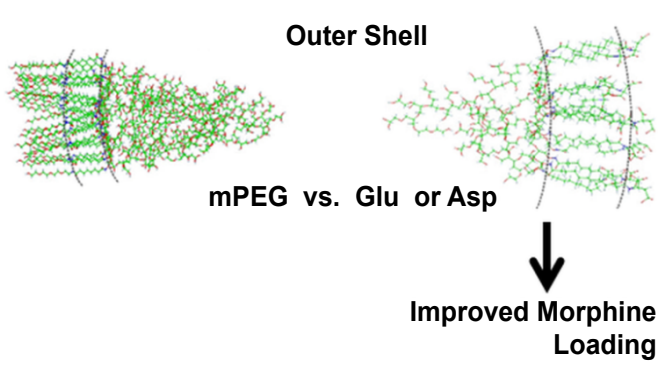

Figure 1: Graphical abstract.

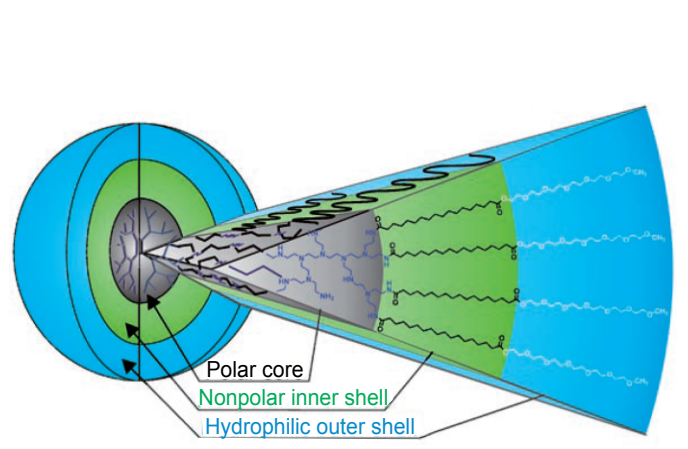

A

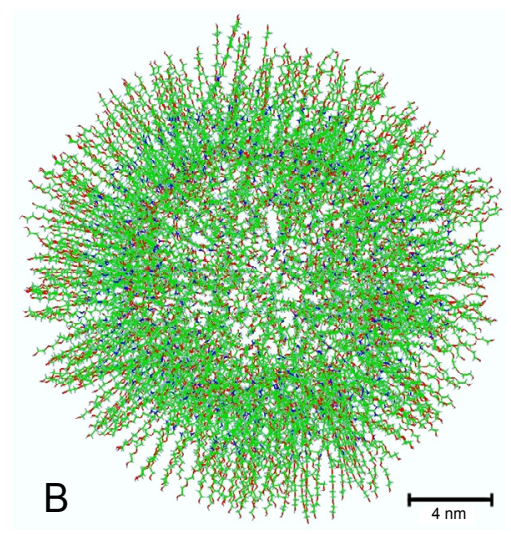

outer shell
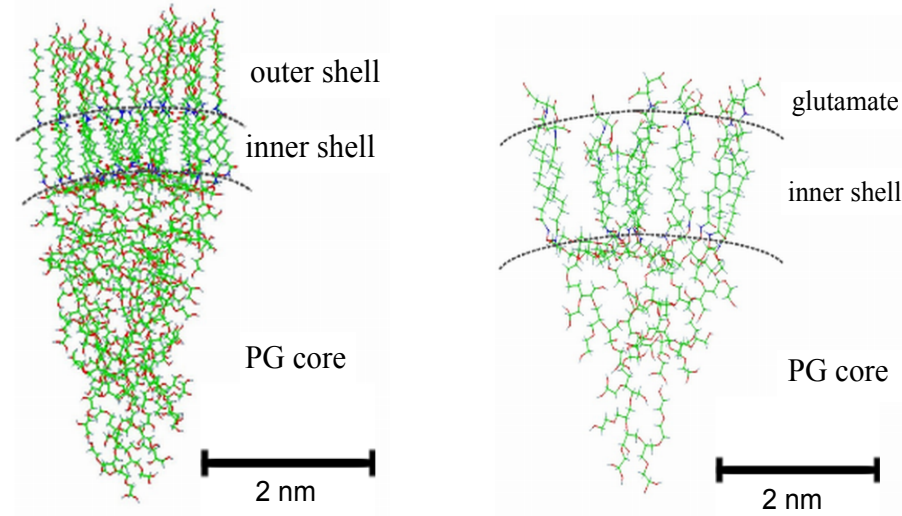

C

$\mathrm{D}$

Figure 2: Structure of the CMS nanotransporter unimer. A, Scheme showing the dendritic polyglycerol amine (polar core), the alkyl chain (nonpolar inner shell) and the monomethoxy poly(ethylene glycol) (mPEG, hydrophilic outer shell). B, Total structure of the chemical available nanotransporter (polar core: radius 3-5 $\mathrm{nm}$, inner shell 3-5 nm, outer shell 3-4 nm). C, Cone-shaped substructure of the simplified nanotransporter with mPEG outer shell (core radius $2 \mathrm{~nm}$, inner shell and outer shell 1.5-2 nm, respectively). D, Cone-shaped substructure of the optimized nanotransporter with a glutamate outer shell (core radius $1-2 \mathrm{~nm}$, inner shell radius $1.5-2 \mathrm{~nm})$.

model building $[11,12]$ resulted in a CMS nanotransporter about 12$13 \mathrm{~nm}$ in size. With MD simulations in an aqueous environment, size declined to 8-9 $\mathrm{nm}$ (Figure 2B, 2C) due to coiling of the long-chain substituents resulting in a shrinking of the shells which is well in line with the size of 7-16 nm derived by dynamic light scattering and CryoTransmission Electron Microscopy [3,13,14]. Next, we simplified the model and came up with a reduced polyglycerol amine core consisting of only 50 monomers (radius $2 \mathrm{~nm}$ ) and alkyl chains containing seven to 14 carbon atoms (radius 1.5-2 nm). In addition, the length of the mPEG chain was reduced to four ethylene glycol repeating units (radius 1.5$2 \mathrm{~nm}$ ). Based on this initial atomistic model of CMS nanotransporters, water molecules were inserted into the simulation box. In the widely used TIP3P solvent model [15] three partially charged atoms (explicit solvent) represent one water molecule $[11,12,16]$. These calculations already proved useful for the investigation of low molecular weight, dendritic micelles with benzol as a guest molecule [17].

\section{Estimation of the preferential localization of guest molecules}

Guest molecules were chosen due to their spatial relations and physicochemical properties, relevant for loading to CMS nanotransporters. We calculated length-to-width-ratios ([18], Figure 3) for planar Congo red $(2.4, \log P$ 0.7), Nile red $(3.2, \log P$ 3.4), and 


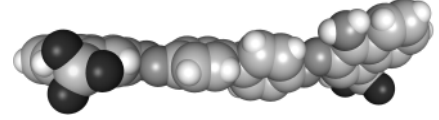

A

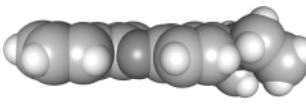

B

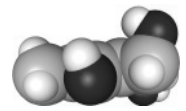

C

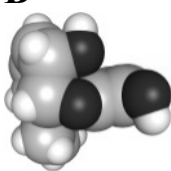

D

Figure 3: Three-dimensional structure of $A$, Congo red $B$, Nile red $C$, pyridoxine and $\mathrm{D}$, morphine. Length-to-width-ratios around 1 indicate globular shape.

\begin{tabular}{|c|c|c|c|c|c|c|c|c|c|}
\hline Cargo & \multicolumn{3}{|c|}{ Nile red } & \multicolumn{3}{c|}{ Morphine } & \multicolumn{3}{c|}{ Morphine- ${ }^{+}$} \\
\hline Compartment & $\Delta \mathbf{G}$ & $\Delta \mathbf{H}$ & $\mathbf{- T} \Delta \mathbf{S}$ & $\Delta \mathbf{G}$ & $\Delta \mathbf{H}$ & $-\mathbf{T} \Delta \mathbf{S}$ & $\Delta \mathbf{G}$ & $\Delta \mathbf{H}$ & $-\mathbf{T} \Delta \mathbf{S}$ \\
\hline Outer Shell & 31.3 & 31.5 & -0.2 & 51.7 & 52.2 & -0.5 & 43.4 & 43.8 & -0.3 \\
\hline Inner Shell & 29.5 & 29.8 & -0.2 & 41.6 & 41.9 & -0.3 & 44.5 & 44.7 & -0.2 \\
\hline Core & 31.2 & 31.4 & -0.2 & 48.2 & 48.7 & -0.4 & 42.8 & 43.2 & -0.3 \\
\hline
\end{tabular}

Table 1: Energy differences $(\mathrm{kJ} / \mathrm{mol})$ for guest molecules in CMS nanotransporters with $\mathrm{mPEG}$ outer shell. Lower differences in free energy $(\Delta \mathrm{G})$, inner energy $(\Delta \mathrm{H})$ and entropy $(\mathrm{T} \Delta \mathrm{S})$ indicate higher loading in the compartments of the simplified CMS nanotransporters.
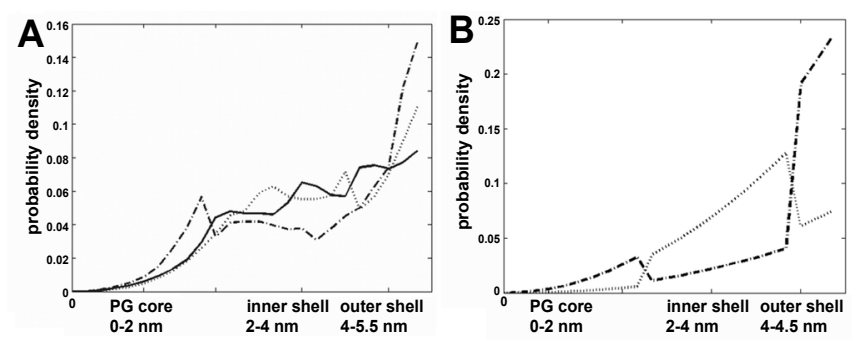

Figure 4: Probability density of guest molecules in simplified CMS nanotransporters with $A, m P E G$ outer shell or B, with Glu outer shell. The curves show the most probable position of the guest molecules (dotted line: Nile red, solid line: morphine, dash-dotted line: morphine- $\mathrm{H}+$ ) inside the CMS nanotransporters once they are loaded (conditional probability, total probability =1).

pyridoxine (2.4, $\log P-0.8)$ as well as globular morphine (1.5, $\log P \leq$ 0.8 ).

Simulations have been started localizing Nile red, morphine (log $P 0.8$ ) and morphine- $\mathrm{H}+$ at a distance of 5-7 $\mathrm{nm}$ of the different parts of the simplified CMS nanotransporters (polar outer shell, nonpolar inner shell and polyglycerol amine core). The reduced sizes of core and shell still exceed the dimensions of the guest molecules and thus will not interfere with the data validity. Before starting the localized MD simulations, the potential energy has been minimized for 20,000 steps and position restrains have been included for all bonds of the structure with 50,000 steps at a temperature of $300 \mathrm{~K}\left(27^{\circ} \mathrm{C}\right)$ and a pressure of 1.0 bar in order to equilibrate the system. Next, MD simulations have been started with 2,000,000 time steps for $4 \mathrm{~ns}$ in total. Localized thermodynamic quantities derived from the simulation data indicate cargo-carrier interactions and thus the most probable location of guest molecules inside the simplified CMS nanotransporter. The probability to find the guest molecule at a certain position inside the nanoparticle is connected to its free energy differences.

Free energy differences $\Delta \mathrm{G}$ were computed by several localized simulations of the guest molecules along a one-dimensional axis from the outer shell via the inner shell into the core according to equation (1) with $\Delta \mathrm{H}$ (inner energy), $\Delta \mathrm{S}$ (entropy) and $\mathrm{T}$ (temperature, $300 \mathrm{~K}$ ).

$$
\Delta G=\Delta H-T \Delta S
$$

$\Delta \mathrm{H}$ mainly reflects hydrophobic or hydrophilic interactions, which can be estimated from Coulomb and Lennard-Jones energies between the guest molecule and its chemical environment [19]. $\Delta \mathrm{H}$ is derived from molecular simulations by taking the mean over stabilizing interactions between the carrier and the guest molecule as provided by Gromacs [11]. The missing quantity $\Delta S$ is derived by a Monte-Carlo quadrature method applied directly to molecular localized thermostated simulation data in the context of the explained simulation strategy [20]. The baseline for computing energy differences are the thermo dynamical quantities of the guest molecules surrounded by solvent only (without CMS nanotransporters) [21]; the respective simulation time has been set to $5 \mathrm{~ns}$. All thermo dynamical quantities given in the results section are understood as differences according to the baseline data (Table 1).

The probability density was obtained at each position of this radius by the Boltzmann exponential of $\Delta \mathrm{G}$ according to equation (2) with $\beta$ being the inverse of temperature times Boltzmann constant and with $\mathrm{w}_{\mathrm{i}}$ being the probability for state $i$.

$$
\Delta G=-\frac{1}{\beta} \ln \left(\frac{w_{1}}{w_{2}}\right)
$$

Yet, along this one-dimensional axis the total volume (with the same molecular properties) differs between core, inner shell, and outer shell. The estimated probability density along the radius simulated by $\mathrm{MD}$ simulations (Figures $4 \mathrm{~A}$ and $4 \mathrm{~B}$ ) represents the conditional probability for the guest molecule to be at a certain position, assuming it is already loaded to the CMS nanotransporter.

\section{Experimental evaluation of morphine loading to CMS nanotransporters}

Using 70\% aminated polyglycerol synthesized as described [22], CMS nanotransporters were produced [3]. CMS nanotransporters were dissolved in $\mathrm{D}_{2} \mathrm{O}$ resulting in a stock solution with a concentration of $5 \mathrm{~g} / \mathrm{L}$. Following the addition of $1 \mathrm{~mL}$ CMS nanotransporter stock solution to morphine $(2.3 \mathrm{mg}, 8 \mu \mathrm{mol} / \mathrm{L})$ the mixture was stirred for $24 \mathrm{~h}$ at room temperature $(1,200 \mathrm{rpm})$ and then filtered through a $0.45 \mu \mathrm{m}$ regenerated cellulose syringe filter for DOSY-NMR experiments (Bruker AV $700 \mathrm{MHz}$ spectrometer, Bruker, Karlsruhe, Germany).

\section{Results and Discussion}

To the best of our knowledge, this study is the first approach to transfer MD simulations to CMS nanotransporters. All compounds investigated are well-defined in structure. Modeling of the structure of the CMS nanotransporter is possible and the result (Figure 2) is in accordance with experimental data $[3,6,15]$. The interaction of Nile red with the chemical available CMS nanotransporters is well-known from experimental investigations [6], too. Morphine loading was not yet tested experimentally. Using the established loading procedure described above, we next checked morphine loading to the CMS nanotransporters by DOSY-NMR experiments.

\section{Preferential localization of Nile red, morphine-H+, and morphine}

UV/Vis spectra, parelectric spectroscopy [6] and fluorescence lifetime imaging [14] have shown that Nile red has a good affinity to CMS nanotransporters composed of a polyglycerol amine core, an 


\begin{tabular}{|c|c|c|c|c|c|c|c|}
\hline \multirow{2}{*}{\multicolumn{2}{|c|}{\begin{tabular}{|c|} 
Cargo \\
Compartment \\
\end{tabular}}} & \multicolumn{3}{|c|}{ Nile red } & \multicolumn{3}{|c|}{ Morphine- $\mathrm{H}^{+}$} \\
\hline & & \multirow{2}{*}{$\begin{array}{c}\Delta \mathbf{G} \\
17.0\end{array}$} & \multirow{2}{*}{$\begin{array}{c}\Delta \mathbf{H} \\
17.1\end{array}$} & \multirow{2}{*}{$\begin{array}{l}-\mathbf{T} \Delta \mathbf{S} \\
-0.1\end{array}$} & \multirow{2}{*}{$\begin{array}{c}\Delta \mathbf{G} \\
15.2\end{array}$} & \multirow{2}{*}{$\begin{array}{c}\Delta \mathbf{H} \\
15.3\end{array}$} & \multirow{2}{*}{$\begin{array}{r}-\mathbf{T} \Delta \mathbf{S} \\
-0.1\end{array}$} \\
\hline \multirow{3}{*}{$\frac{2}{0}$} & Outer Shell & & & & & & \\
\hline & Inner Shell & 14.9 & 15.0 & -0.1 & 18.8 & 19.0 & -0.3 \\
\hline & Core & 18.8 & 19.0 & -0.2 & 15.6 & 15.8 & -0.1 \\
\hline \multirow{3}{*}{$\frac{\circ}{\frac{9}{\alpha}}$} & Outer Shell & 15.9 & 16.0 & -0.1 & 13.4 & 13.4 & -0.1 \\
\hline & Inner Shell & 12.5 & 12.6 & -0.9 & 18.9 & 19.2 & -0.2 \\
\hline & Core & 16.1 & 16.3 & -0.2 & 14.7 & 14.8 & -0.1 \\
\hline
\end{tabular}

Table 2: Energy differences $(\mathrm{kJ} / \mathrm{mol})$ for guest molecules in CMS nanotransporters with glutamate (Glu) and aspartate (Asp) outer shell. Lower differences in free energy $(\Delta G)$, inner energy $(H)$, and entropy $(T \Delta S)$ indicate higher loading in the compartments of the optimized CMS nanotransporters.

inner alkyl shell and an outer mPEG shell. This can also be seen in computer simulations, the differences in free energy $(\Delta \mathrm{G})$, inner energy $(\Delta \mathrm{H})$ and entropy $(\Delta \mathrm{S})$ of Nile red for the different shells are itemized in Table 1. The lower the free energy difference, the stronger the interaction of the CMS nanotransporter and the guest molecule [23]. While values in Table 1 give an average of free energy differences for each compartment of CMS nanotransporters, Figure 4 depicts detailed probability densities for the location of guest molecules within the CMS nanotransporter compartments (if the guest molecules are loaded to the CMS nanotransporter).

Localized thermodynamic simulations indicate that Nile red tends to stay in the inner shell of simplified CMS nanotransporters (Table 1), a slight preference for the inner shell was seen by fluorescence measurements [2], too, which also enabled for first insights into the mechanism for penetration enhancement by dendrimeric nanoparticles. Distribution of Nile red within the nanotransporter shells as well as the flexibility of the polymer branches in the unimolecular state of the nanotransporter depend on the temperature. The increasing space for the mobility of the incorporated Nile red and a higher probability density (Figure 4) of cargo-solvent interactions at temperatures above $304 \mathrm{~K}\left(31^{\circ} \mathrm{C}\right)$ indicate the potential of the CMS nanotransporter for drug delivery to the skin in the critical temperature range - the skin surface temperature is $305 \mathrm{~K}\left(32^{\circ} \mathrm{C}\right)$. Yet, the loaded CMS nanotransporters can retain their cargo and are capable to transport [14] to the stratum corneum where various nanoparticles firmly adhere [24]. This may improve skin penetration of Nile red to normal, damaged and diseased skin [7]. Moreover, the 8- to 13-fold enhancement of Nile red penetration, induced by CMS nanotransporters [6] and the respective 11-fold enhancement of the polar dye Rhodamin B [5] indicate that the properties of the nanocarrier are most important and may exceed the relevance of the intrinsic penetrability of the loaded agent.

Next, we studied the interaction of the globular and polar morphine $(\log P \leq 0.8)$ and the simplified CMS nanotransporter. Differences in free energy of protonated and non-protonated morphine exceed those of Nile red more than $10 \mathrm{~kJ} / \mathrm{mol}$ (Table 1 ). The thereby indicated poor morphine loading was proven by DOSY-NMR results (data not shown). In fact, morphine clearly differs from the well-loadable compounds by shape (Figure 3). Yet, once a morphine molecule is taken up, the maximum of the probability density of protonated morphine along a one-dimensional axis indicates a preference of the opioid's protonated amino group towards the amide-linker, attaching the inner shell to the particle core. Thus, Nile red and non-protonated morphine have a similar probability density (once they are loaded), whereas the inner shell appears to be a less favorable location for protonated morphine (Figure 4A).

The experimental loadings, obtained for Nile red and morphine, proved the computational approach based on the cone-shaped substructure. This indicates the relevance of shape of a guest compound for cargo-carrier interaction. However, more complex calculation approaches are needed to address the physiological environment and the aggregation of the CMS nanotransporters unimers. Moreover, a cut-off-value for the discrimination of poor or high loading has not yet been identified. Cut-off-values need to be determined in large scale studies with statistical relevant numbers of guest compounds and should facilitate decision making in simulation-based carrier development.

\section{CMS nanotransporters with higher affinity to morphine-H+}

Next, we aimed to improve CMS nanotransporters for morphine loading. The mPEG outer shell was replaced by aspartate (Asp) or glutamate $(\mathrm{Glu})$, respectively. The acidic surface of the optimized nanoparticles should bind protonated morphine by electrostatic forces. Nanoparticle size decreased to 5-7 nm (Figure 2D), free energy difference for loaded morphine- $\mathrm{H}+$ declines from about $43 \mathrm{~kJ} / \mathrm{mol}$ (Table 1) to less than $16 \mathrm{~kJ} / \mathrm{mol}$ (Table 2; Glu) or less than $14 \mathrm{~kJ} / \mathrm{mol}$ (Table 2; Asp) with the outer shell and the entropy difference is clearly enhanced. Free energy differences as well as probability densities (Figure 4B) revealed improved cargo-carrier interactions and the preferential location of morphine-H+ in the outer shell of optimized CMS nanotransporters. Morphine binding by electrostatic forces could retard opioid release. Considering the modelled timescale (in the range of nanoseconds), we did not calculate release rates. A potential retarded morphine release could contribute to improved pain control by topical morphine therapy in burned patients and following split skin removal for grafting. Currently, this approach is of limited efficacy [25]. Release studies and clinical trials will test this hypothesis. Importantly, morphine also improves wound healing due to enhanced keratinocyte migration [10].

Importantly, by changing the outer shell, the optimized CMS nanotransporter can still take up lipophilic guest molecules like Nile red. Compared to the CMS nanotransporter with the PEG outer shell, differences in free energy of loaded Nile red was reduced by half: around $30 \mathrm{~kJ} / \mathrm{mol}$ (Table 1) compared to around $15 \mathrm{~kJ} / \mathrm{mol}$ (Table 2, Glu) and $13 \mathrm{~kJ} / \mathrm{mol}$ (Table 2, Asp) [24,25]. The inner shell, relevant for Nile red incorporation into the nanotransporter, is still given when replacing the $\mathrm{mPEG}$ outer shell with aspartate or glutamate. Thus, the optimized nanotransporter may be at least as versatile as the chemical available type. Currently, the local tolerability of the optimized CMS nanotransporters remains unknown. Yet this carrier lacks a cationic surface - a frequent reason for poor tolerability of dendrimers [26].

Due to the molecular assembly of CMS nanotransporters, the glutamate or aspartate can be easily connected via amide bounds to the inner shell. To retain the penetration enhancing effects of the CMS nanotransporters when applied to human skin [5-7] and the good tolerability [4], we suggest an incomplete replacement of mPEG by glutamate or aspartate.

Our study substantiates major differences in the cargo-carrier interactions of planar (e.g. Nile red) and globular (e.g. morphine) guest molecules. Space between the alkyl or mPEG molecules should limit the loading of voluminous cargos. An easy accessible cargo binding to the carrier surface should overcome this problem. This is well in accordance with pyridoxine reducing the size of loaded CMS nanotransporters to approximately $20 \mathrm{~nm}$ diameter without the formation of larger CMS nanotransporters aggregates. Planar, polar molecules like Congo red showed a deeper incorporation into the carrier [3]. Moreover temperature and solvent depending cargo-carrier interactions [14] e.g. in the range of skin surface temperature which is $32{ }^{\circ} \mathrm{C}$ with normal 
Citation: Weber M, Zoschke C, Sedighi A, Fleige E, Haag R, et al. (2014) Free Energy Simulations of Cargo-Carrier Interactions for Core-Multishell Nanotransporters. J Nanomed Nanotechnol 5: 234. doi: 10.4172/2157-7439.1000234

skin yet increases with inflamed skin may allow to adapt a carrier to disease related needs.

\section{Conclusion}

Free energy simulations describe the structure of the chemically well-defined CMS nanotransporters and predict drug incorporation and attachment to the particle's surface. Our hands-free computational approach proved applicable to predict cargo-carrier interactions of two model compounds and adds an interesting approach to the long-term goal of efficient preclinical drug development. Moreover, we propose an optimized CMS nanotransporter with enhanced loading of the globular, polar cargo morphine.

\section{Acknowledgement}

This work was financially supported by the German Research Foundation (DFG, project A02 to R.H., and project C02 to M.SK. of SFB1112) and by the Freie Universität Berlin (Focus Area Nanoscale, R.H., M.SK.). Christian Zoschke gratefully acknowledges a doctoral scholarship of German National Academic Foundation (Studienstiftung des deutschen Volkes e.V.).

\section{References}

1. Haag R (2004) Supramolecular drug-delivery systems based on polymeric core-shell architectures. Angew Chem Int Ed Engl 43: 278-282.

2. Quadir MA, Radowski MR, Kratz F, Licha K, Hauff $P$, et al. (2008) Dendritic multishell architectures for drug and dye transport. J Control Release 132: 289294.

3. Radowski MR, Shukla A, von Berlepsch H, Böttcher C, Pickaert G, et al. (2007) Supramolecular aggregates of dendritic multishell architectures as universal nanocarriers. Angew Chem Int Ed Engl 46: 1265-1269.

4. Wolf NB, Küchler S, Radowski MR, Blaschke T, Kramer KD, et al. (2009) Influences of opioids and nanoparticles on in vitro wound healing models. Eur J Pharm Biopharm 73: 34-42.

5. Küchler S, Abdel-Mottaleb M, Lamprecht A, Radowski MR, Haag R, et al. (2009) Influence of nanocarrier type and size on skin delivery of hydrophilic agents. Int J Pharm 377: 169-172.

6. Küchler S, Radowski MR, Blaschke T, Dathe M, Plendl J, et al. (2009) Nanoparticles for skin penetration enhancement--a comparison of a dendritic core-multishell-nanotransporter and solid lipid nanoparticles. Eur J Pharm Biopharm 71: 243-250.

7. Alnasif N, Zoschke C, Fleige E, Brodwolf R, Boreham A, et al. (2014) Penetration of normal, damaged and diseased skin--an in vitro study on dendritic coremultishell nanotransporters. J Control Release 185: 45-50.

8. Bujotzek A, Weber M (2009) Efficient simulation of ligand-receptor binding processes using the conformation dynamics approach. J Bioinform Comput Biol 7: 811-831.

9. Durmaz V, Schmidt S, Sabri P, Piechotta C, Weber M (2013) Hands-of linear interaction energy approach to binding mode and affinity estimation of estrogens. J Chem Inf Model 53: 2681-2688.
10. Heilmann S, Küchler S, Wischke C, Lendlein A Stein C et al. (2013) A thermosensitive morphine-containing hydrogel for the treatment of large-scale skin wounds. Int J Pharm 444: 96-102.

11. Van Der Spoel D, Lindahl E, Hess B, Groenhof G, Mark AE, et al. (2005) GROMACS: fast, flexible, and free. J Comput Chem 26: 1701-1718.

12. Lindahl E, Hess B, van der Spoel D (2001) GROMACS 3.0: a package for molecular simulation and trajectory analysis. J Molecular Modeling 7: 306-317.

13. Fleige E, Ziem B, Grabolle M, Haag R, Resch-Genger U (2012) Aggregation Phenomena of Host and Guest upon the Loading of Dendritic Core-Multishell Nanoparticles with Solvatochromic Dyes. Macromolecules 45: 9452-9459.

14. Boreham A, Pfaff M, Fleige E, Haag R, Alexiev U (2014) Nanodynamics of dendritic core-multishell nanocarriers. Langmuir 30: 1686-1695.

15. Takemura K, Kitao A (2007) Effects of water model and simulation box size on protein diffusional motions. J Phys Chem B 111: 11870-11872.

16. Tavagnacco L, Schnupf U, Mason PE, Saboungi ML, Cesàro A, et al. (2011) Molecular dynamics simulation studies of caffeine aggregation in aqueous solution. J Phys Chem B 115: 10957-10966.

17. Tyagi R, Malhotra S, Thünemann AF, Sedighi A, Weber M, et al. (2013) Investigations of Host-Guest Interactions with Shape-Persistent Nonionic Dendritic Micelles. The Journal of Physical Chemistry C 117: 12307-12317.

18. Sousa da Silva AW, Vranken WF (2012) ACPYPE - AnteChamber PYthon Parser interfacE. BMC Res Notes 5: 367.

19. Jain T, Jayaram B (2005) An all atom energy based computational protocol for predicting binding affinities of protein-ligand complexes. FEBS Lett 579: 66596666 .

20. Weber M, Andrae K (2010) A simple method for the estimation of entropy differences. Comm Math Comp Chem 63: 319-332.

21. Klimm M, Bujotzek A, Weber M (2011) Direct reweighting strategies in conformation dynamics. Match-Comm Math Comput Chem 65: 333-346.

22. Roller S, Zhou H, Haag R (2005) High-loading polyglycerol supported reagents for Mitsunobu- and acylation-reactions and other useful polyglycerol derivatives. Mol Divers 9: 305-316.

23. Böhm HJ (1996) Current computational tools for de novo ligand design. Curr Opin Biotechnol 7: 433-436.

24. Baroli B (2010) Penetration of nanoparticles and nanomaterials in the skin fiction or reality? J Pharm Sci 99: 21-50.

25. Stein C, Küchler S (2012) Non-analgesic effects of opioids: peripheral opioid effects on inflammation and wound healing. Curr Pharm Des 18: 6053-6069.

26. Jain K, Kesharwani P, Gupta U, Jain NK (2010) Dendrimer toxicity: Let's mee the challenge. Int J Pharm 394: 122-142. 